\title{
Effective energy storage power control to extend the acceptable range of generator frequency in distributed generation
}

\author{
Pavel Ilyushin ${ }^{1, *}$, Sergey Filippov ${ }^{1}$, and Aleksandr Kulikov ${ }^{2}$ \\ ${ }^{1}$ Energy Research Institute of Russian Academy of Sciences, Nagornaya str., 31, bld. 2, Moscow, Russia \\ ${ }^{2}$ Nizhny Novgorod State Technical University n.a. R.E. Alekseev, Minin str., 24, Nizhny Novgorod, Russia
}

\begin{abstract}
Gas-turbine and gas-piston generator sets (GS) are widely used at distributed generation (DG) facilities. International GS manufacturers configure relay protections (RP) in a way that narrows the acceptable range (AR) and causes properly functioning RPs to effect unnecessary disconnections. It has been shown that the worst disturbances are observed when a microgrid that carries a DG facility is islanded, and power imbalance occurs; when motors start in groups; when the GS's shed 95\% of their output; and when a 3-phase short-circuit occurs in an islanded grid segment. Energy storage units (ESU) are the most recent and highly successful solution to many problems of power engineering. The authors hereof have developed a method that controls active and reactive power of an ESU independently to avoid unnecessary GS disconnections that would otherwise be caused by a short-term deviation in frequency; this will help reliably deliver electricity to the users of an islanded microgrid. Simulation results show that the use of ESUs helps to effectively extend the acceptable range for generator sets. There are recommendations on drawing technical requirements to ES. An important advantage of the proposed ESU power control method is that it does not need to adjust ES-received control actions (CA) online for frequency deviation.
\end{abstract}

\section{Introduction}

International practices have shown that distributed generation (DG) develops worldwide mainly in the form of constructing renewable energy (RE) facilities. Experts project RE will account for $27.1 \%$ of global electricity generation by $2030,48.8 \%$ by 2050 . Wind farms will be the most common type $(\sim 70 \%)$ of RE facilities in 2030 . However, by 2050 they will drop to $47 \%$ due to the rising popularity of solar farms, as high-efficiency photovoltaic modules will be getting cheaper [1-3].

In Russia, DG facilities are mostly constructed by the owners of industrial facilities and use non-renewable energy (hydrocarbons). As a rule, such facilities are based on gas-turbine units (GTU), gas-piston units (GPU), and diesel-fired generator sets (GS), all of which are imported.

These GS's are equipped with relay protections (RP), automatic speed controls (ASC), and automatic excitation controls (AEC), all of which are compliant with international / foreign standards, which determines the quality and parameters of transients.

One manufacturer's GPU documentation specifies RP settings that will trigger a disconnection if the grid frequency is $>51.5 \mathrm{~Hz}$ or $<49 \mathrm{~Hz}$ for $0.2 \mathrm{~s}$. Another GPU manufacturers configure their units to disconnect if the frequency exceeds $55 \mathrm{~Hz}$ for 4 seconds or drops below $47.5 \mathrm{~Hz}$ for $2 \mathrm{~s}$ [4-6].

When the microgrid that carries a DG facility becomes islanded and has an active power excess / deficit, or when disturbances occur in an microgrid, short-term frequency deviations may occur that will cause GS's to disconnect. Cases have been reported where GS's were disconnected due to disturbances in an external distribution grid that the microgrid was synchronized with; or within the microgrid, where RPs were functioned correctly as configured. This approach to RP configuration on the part of international manufacturers narrows the GS acceptable range (AR), which in case of industrial processes may lead to an accident or an emergency [7-9].

Energy storage units (ESU) are the latest and highly successful solution to various problems of power engineering, and they have found broad use in power systems around the world [10].

The authors hereof propose the method that controls active and reactive power of an ESU independently to avoid unnecessary frequency deviation-triggered GS disconnections in case of emergency or post-emergency operation. The paper shows calculated transients for islanded operation of a DG facility-carrying microgrid; these calculations prove the proposed method to be effective and efficient, as it helps to minimize user damage even in case of a severe disturbance.

\footnotetext{
* Corresponding author: $\underline{\text { ilyushin.pv@mail.ru }}$
} 


\section{Energy storage practices for power systems}

Consider how an ESU equipped with a highperformance automatic control system (ACS) could be used to provide power in emergency or post-emergency operation, where a transition to independent active/reactive power control could be deemed instantaneous in the first approximation.

Whether using an ESU is feasible or not shall be determined by feasibility testing of the available options given how costly but efficient ESUs are; ESUs are applicable where an industrial facility has high requirements to the reliability of electricity delivery to the critical users, and disruptions are too damaging.

Since short-term disruptions are most common, the effectiveness of using an ESU in conjunction with other emergency measures shall be determined by analyzing the statistics on the occurrence rates of such disruptions and their associated damage [11-13].

In case of islanded operation of DG facilities, ESUs are recommendable when:

- The DG facility is subject to emergency outage, in which case an ESU will be running until normal electricity delivery has been restored, or until the production process can be stopped without causing an emergency,

- An ESU will help to relieve local overloads in the microgrid exposed to maximum loads, e.g. when weather changes abruptly, the equipment is subject to emergency shutdown, etc.,

- An ESU is to be used as a backup power source to start a DG facility "from scratch" after it has been unexpectedly shut down due to an external grid accident,

- An ESU will be needed to deliver electricity to critical users while addressing a short circuit or when automatic reclosers or automatic load transfer (ALT) devices included in dynamic voltage sag/interruption compensators have the deadtime,

- It will help to prevent a voltage collapse in case the microgrid that carries multiple high-power motors is exposed to a substantial power deficit.

When adjusting the control actions (CA) for ESUs, one should bear the following in mind:

- When arranging emergency controls for for islanded operation of ESU-equipped DG facilities, it is crucial to optimize the distribution of control actions between different controlled facilities,

- Executing a control action to cause the ESU to deliver active power is not advisable if frequency deviations are insignificant and short,

- If an SC occurs near the electricity users' busbars, ESUs will not be able to normalize voltage, as that will require far greater power. However, electricity delivery can be improved by shortening the time it takes to address the SC,

- When restoring motors to normal operation after a short-term SC, one needs to pay attention to transients in synchronous motors (SM). They can be destabilized even by a short-term voltage sag, especially if an SM is running while ALTs with $\cos \varphi \approx 1$ are offline.
Asynchronous SM operation can get too long, causing significant ripple. The following emergency measures could be effective: SM field suppression for easier resynchronization; disconnecting from the grid if the chances for resynchronization are slim,

- CA adjustments should be made by calculating the transients and be guaranteed to have an actual impact.

Let us analyze the feasibility of using a combined supercapacitor + li-ion battery ESU to extend the AR of a GS running in a DG facility. International practices have shown that solution not only optimizes the costs but also covers the required power output/consumption rates and durations (up to 10-30 s).

Supercapacitors are enhanced capacitors that use constant voltage and have a high charge density thanks to the design and special treatment of electrodes, which makes supercapacitors far longer-lasting than their conventional counterparts. With their extremely large capacitance on the order of several farads, supercapacitors can store substantial energy and deliver it when needed in the form of high currents. At the same time, supercapacitors are classified as shot-term energy storage; however, they are small and low-maintenance.

High-power batteries are usable in power engineering as ESUs to compensate non-steady electricity generation by RE facilities, to control power flows over power transmission lines (PTL), or to reduce the amplitude of power fluctuations. The most common types are leadacid, nickel-cadmium, and lithium-ion batteries.

The proposal here is to connect an ESU to the generator voltage busbars of the DG facility via a dualbridge reversible converter (rectifier-inverter). One common solution is reversible three-phase converters based on semiconductor power rectifiers with buffer gates in the zero wire; these units can function as rectifiers or inverters depending on the control angles. To solve the problem, we need biparametric ESU power control, which means it should use two parameters (active and reactive power) and be able to control them independently. In that case, the converter independently controls separate gate groups.

To implement biparametric control over ESU power output and ESU power consumption, the ESU may use one of several circuitries, e.g. a six-phase circuit with a zero output and an interphase transformer, a bridgebased circuit, etc.; these circuitries utilize bioperational (fully controlled or locking) gates capable of switching high currents at 6 to $20 \mathrm{kV}$, e.g. state-of-the-art insulated-gate bipolar transistors (IGBT) and MOS transistors.

Gate switching in a converter induces higher harmonics in the microgrid, whereby the interference level depends on the magnitude of switched current. Higher harmonics are known to negatively affect virtually any kind of primary and secondary equipment. This is why when connecting an ESU to a microgrid, power engineers need to find out how the operating parameters of a semiconductor converter will affect the voltage sinewave distortion in ESU-grid power transfer; this will help to determine if harmonic filters are needed. 


\section{$3 \quad$ Preventing disconnections}

unnecessary

GS

A DG facility may be required to sustain an infinitely long islanded operation depending on the microgrid-togrid PTL condition and/or the conditions of power transformers, the operating parameters of the grid/microgrid, the staff-on-duty's decisions, etc. In such operating situations, there are several things to know about electricity delivery from generator sets at DG facilities.

Any operating situation of an islanded DG facility is either of the two cases:

- Voltage early in a transient is low due to $\mathrm{SC}$, or a group of motors is connected with an excessive slip. Motor self-start or starting a group of motors often requires extra generation of reactive power, which should be borne in mind when configuring the ESU,

- Early in a transient, there are no conditions that would cause motors to brake. Voltage and frequency deviations are less significant, and the ASCs/AECs are able to normalize the operating parameters. If for any reason, the operating parameters need to be adjusted to avoid triggering the RPs of the GS, one can effectively use UFLS-1 systems backed up by ESUs.

The first case is when an ALT is triggered with 2 to 3 second of deadtime, in which case having the ESU provide all the necessary reactive power is neither technically or economically feasible. The advisable course of action here is to execute a load-shedding (LS) $\mathrm{CA}$ and use special automations to launch small motor groups consecutively while monitoring busbar voltages; or use smooth starters and variable frequency drives.

In the second case, the worst disturbance is when one or more GS's disconnect unexpectedly. Compared to asymmetric GS speed control (where the GPU drive gains power at a lower rate than decreases it), symmetric one is associated with a lesser difference in the quality of transients and in the requirements to $\mathrm{CA}$ adjustments.

If the total available power output of all the connected GS's is not enough to cover the current load, an LS CA should be run by one of the two methods below:

1. Use UFLS-1 with a frequency setpoint above $f_{\text {min }}$ of the GS RPs and load-shedding that total for no less than the nominal power output of the largest generator set at the DG facility.

2. Use an emergency response (ER) system that will $\log$ the GS disconnection and use the previously recorded operating parameters to determine the actual disconnected generator output, which equals the previously recorded GS output, in order to issue a CA that will load-shedding when the frequency setpoint is reached.

Consider an industrial microgrid that carries a DG facility, see Figure 1 for a simplified single-line circuit diagram.

Infinite power buses (IPB) represent all the external sources of an equivalent constant EMF behind a resistance that corresponds to the $\mathrm{SC}$ power. The microgrid carries a total consumption of $20 \mathrm{MW}$, whilst its GPUs have a total capacity of $10 \mathrm{MW}$. Consumption breakdown is as follows: $10 \%$ for SMs, $62 \%$ for asynchronous motors (AM), and $28 \%$ for static load. Equivalent AMs have different parameters: $M_{\text {stat }} / M_{\text {nom }}=$ 0.2 (in AMs constituting $20 \%$ of the total AM-related load), $0.4(70 \%)$, or $0.8(10 \%)$. The total nominal power output of the running GS's varies. CA is implemented on an ESU that is connected to the GS busbars of the DG facility at point $\boldsymbol{C}$, see Figure 1 . Calculations of transients are performed in the software package MUSTANG.

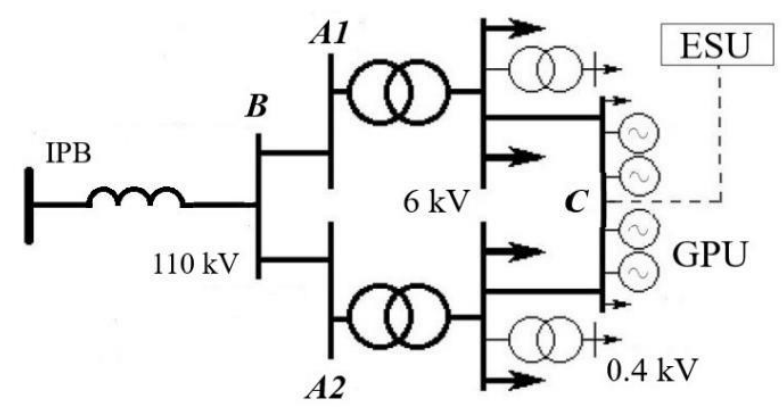

Fig. 1. Simplified single-line circuit diagram of a microgrid carrying a DG facility.

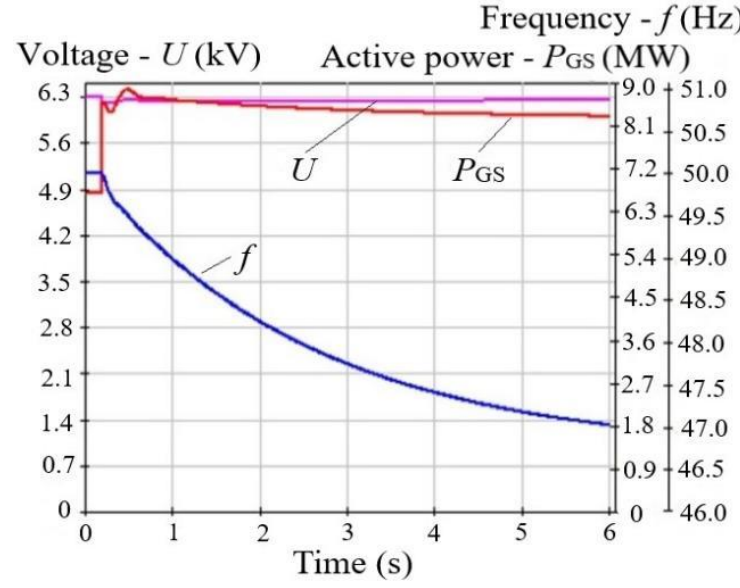

a)

Frequency $-f(\mathrm{~Hz})$

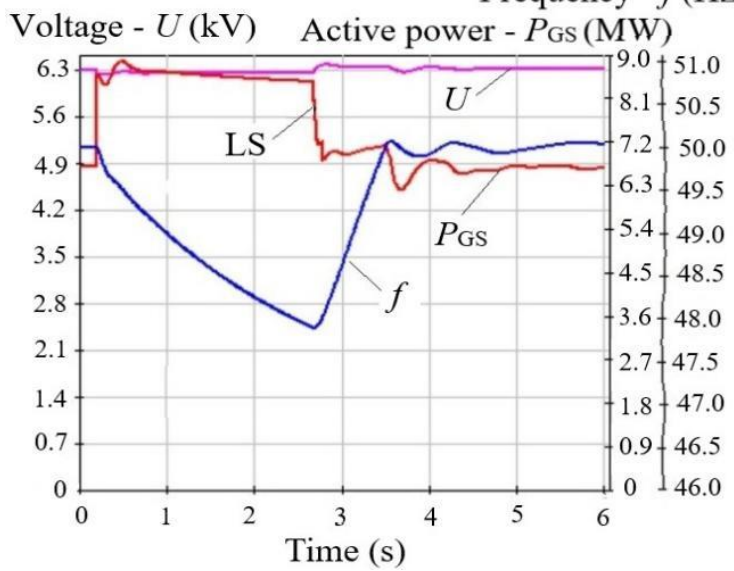

b)

Fig. 2. Transients, a single-GPU disconnection: (a) no CA; (b) ER system-issued adjusted CA triggers load shedding ( $\mathrm{P}_{\mathrm{GS}}$ equals the power output of the three GPUs that are still online).

Figure 2 shows transients associated with SC-free disconnection of a single of four GPUs, $P_{\mathrm{GS} . n o m}=2.5$ 
MW, asymmetric speed control, total load $P_{\text {load }}=9.2$ MW in islanded operation. Before disconnecting, the GPU had been carrying a load of $\approx 2.5 \mathrm{MW}$, the rest carried 2.24 MW each.

Without executing an LS CA (Figure 2a), the microgrid sets at $f=46.7 \mathrm{~Hz}$, whereby the frequency droop $\approx 0$ (the available power output of all the connected GPUs is $110 \%$ ). Once an ER device is triggered (Figure 2b), the GPU is disconnected, and the frequency drops to $f=48 \mathrm{~Hz}$, an LS CA of $2.5 \mathrm{MW}$ is executed stepwise over $0.1 \mathrm{~s}$. Before the frequency drops, the ER action helps to minimize the CA magnitude and keep delivering electricity to the majority of users.

The transient shown in Figure $2 \mathrm{~b}$ might cause other GPUs to disconnect by triggering RPs as voltage drops shortly if the setpoints are close to $U_{\text {nom, and the time }}$ delays are shorter than the configured maximum permissible SC duration in the adjacent grid.

Running an ESU CA to increase the GS busbar voltage for until the SC has been addressed will be costineffective in most cases given the substantial required reactive power $Q_{\mathrm{CA}}$. It is therefore advisable to consider redesigning grid RPs to handle SCs faster and to alter the grid response so that SC-related voltage sags be less deep.

\section{ESUs to extend acceptable frequency range of GS's}

Preventing GS RPs from triggering when the frequency deviates shortly is most important for islanded DG facility-carrying microgrids when mechanical constants of GS inertia are low, especially when GPU speeds are controlled asymmetrically, and the setpoints $f_{\min }, f_{\max }$ are close to $f_{\text {nom }}$ [14-16].

Let us now break down the key points of the proposed ESU control method intended to stabilize the frequency in a microgrid that carries a DG facility:

1. The ESU control method consists in issues appropriate control actions to the ESU when the generator shaft becomes imbalanced, which causes the microgrid frequency to deviate from $f_{\text {nom. The }} \mathrm{CA}$ is adjusted in a way that keeps the current frequency within a narrower range than the range specified by the GS manufacturer in the form of RP setpoints.

2. ESU CAs need to be adjusted differently for the early grid disturbance-caused transient and for the interim points of the transient when the system is not in a steady state.

3. Early in a transient, as a rapid change in generation $\left(\Delta P_{\mathrm{GS}}\right)$ is detected, it is necessary to issue as quickly as possible an active-power ESU CA (CA $A_{\mathrm{ESU}-P}$ equal to $\Delta P_{\mathrm{ESU}}$ ) adjusted to be approximately equal to $\Delta P_{\mathrm{GS}}$. Adjusting $P_{\mathrm{GS}}$ directly will be inefficient as switchings within the microgrid will cause rapidly varying currents: subtransient and aperiodic currents, as well as harmonics attributable to phase asymmetry. Attaining a correct $P_{\mathrm{GS}}$ may involve longer measurements and thus slow down the execution of CAESU-P.
4. For reasons specified in cl. 3, it is reasonable first to execute $\mathrm{CA} \mathrm{ASU}_{\mathrm{ES}} \mathrm{P}$ that is close to $\Delta P_{\mathrm{ESU}}$ in proportion to the derivative of $f$ :

$$
\Delta P_{\mathrm{ESU}}=-K_{d f} \cdot(d f / d t)_{0},
$$

where the proportionality coefficient $K_{d f}$ needs to be precalculated by connecting loads of different magnitudes and recording the increase in the GS load $\left(\Delta P_{0}\right)$ and the derivative $(d f / d t)_{0}$, see Figure 3.

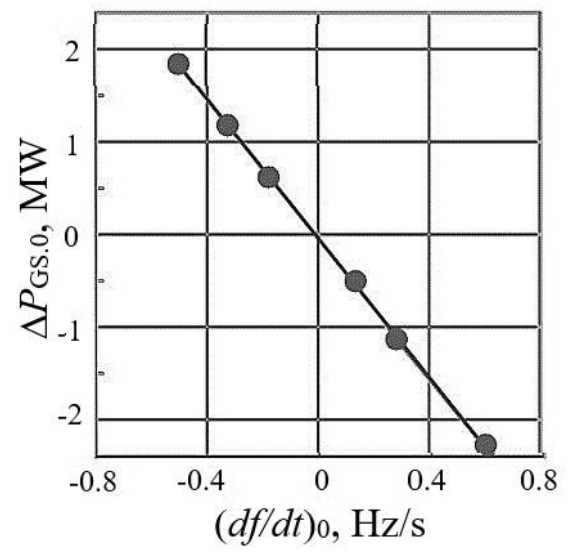

Fig. 3. Curve of $\Delta P_{\mathrm{GS} .0}=\varphi(d f / d t)$ for a DG facility-carrying $\operatorname{microgrid}\left(K_{d f} \approx-3.74 \mathrm{MW} \cdot \mathrm{s} / \mathrm{Hz}\right)$.

5. In a transient caused by load surge, $\Delta P_{\mathrm{GS}}$ and $d f / d t$ cannot be matched one-to-one due to the effects of dynamic power components, which means CAESU-P needs to be executed differently. Boundary frequency values $f_{1}, f_{2}$ should be set for the proposed microgrid frequency control method in addition to $f_{\min }, f_{\max }$, which are the GS RP setpoints, as follows:

$$
f_{\min }<f_{1}<f<f_{2}<f_{\max } .
$$

6. To control the transient, the ESU ACS should keep checking frequency at equal time intervals $T$, and if $f<$ $f_{1}$, execute a CA to raise $P_{\text {ESU }}$ (i.e. lower the GS output and therefore increase the frequency); or, if $f>f_{2}$, lower $P_{\text {ESU. }} T$ and ESU power increments $\left(+\Delta P_{\mathrm{ESU}},-\Delta P_{\mathrm{ESU}}\right)$ should be optimized by simulation [17-20].

7. Controls as described in $\mathrm{Cl}$. 6 can be made more efficient by adjusting the increments $\pm \Delta P_{\mathrm{ESU}}$ in proportion to the current frequency derivative $(d f / d t)_{t}$.

8. Once the controlled part of the transient is over, when for 6-10 seconds, there is no need to adjust $\Delta P_{\mathrm{ESU}}$, i.e. $f_{1}<f<f_{2}$, and $\mathrm{CA}_{\mathrm{ESU}-P}$ is smoothly or incrementally nullified by the ESU ACS, the system should run the ESU charge recovery algorithm.

9. Since when islanded, load surges cause the GS frequency to drop for a short time, this phenomenon should be utilized to somewhat reduce power surging for the ESU, which reduction should be commensurate with the effect that load has on the frequency.

10. ESU reactive power control (CA $\mathrm{CASU}_{\mathrm{E}}$, the output power $\left.\Delta Q_{\mathrm{ESU}}\right)$ can use relay-forced generator excitation: $\Delta Q_{\mathrm{ESU}}>0$, if $U<U_{\min }$, optimize the parameters by simulation.

Let us analyze the computed transients in which the GS successfully remained online while islanded thanks 
to ESU active and reactive power control as described above.

The RPs had the following GPU frequency setpoints: $f_{\min }=47.5 \mathrm{~Hz}, 2$-second delay, $f_{\max }=55 \mathrm{~Hz}, 4$-second delay. To control ESU power, the acceptable range was set as $f_{1}=49, f_{2}=51 \mathrm{~Hz}$. Adjustments were made in the protocol for the fact that the constrain $f_{1}<f<f_{2}$ may be violated for a time shorter than the GPU RP delays. $T$ equals $300 \mathrm{~ms}$ (varying within $200-500 \mathrm{~ms}$ did not seem to affect the effectiveness of this control method); $\Delta P_{\text {ESU }}$ increments were $+10 \%$ and $-20 \%$ of the preceding value.

For simulation modeling, it was assumed that early in a transient, ESU power control was invoked with a 60ms delay (triggering the launcher; computing $(d f / d t)_{0}$ and $\Delta P_{\mathrm{ESU}}$; issuing the CA to the ESU), whilst further controls (based on frequencies below $f_{1}$ or above $f_{2}$ ) were executed with a 30 -ms delay.

Below there are three examples of executing $\mathrm{CA}_{\mathrm{ESU}-P, Q}$ where the method prevented GPU disconnections using the same $\mathrm{CA}$ adjustments in fundamentally different transients that were associated with severe disturbances. The conclusion is that it is not necessary to adjust CAs online by frequency deviation.

\subsection{Load surge due to simultaneous start of an AM sroup}

The conditions for starting three equivalent AMs are the same as above, the GPU load before surging is $30 \%$ of $P_{\mathrm{GS} . n o m}$, the transient is shown in Figure 4.

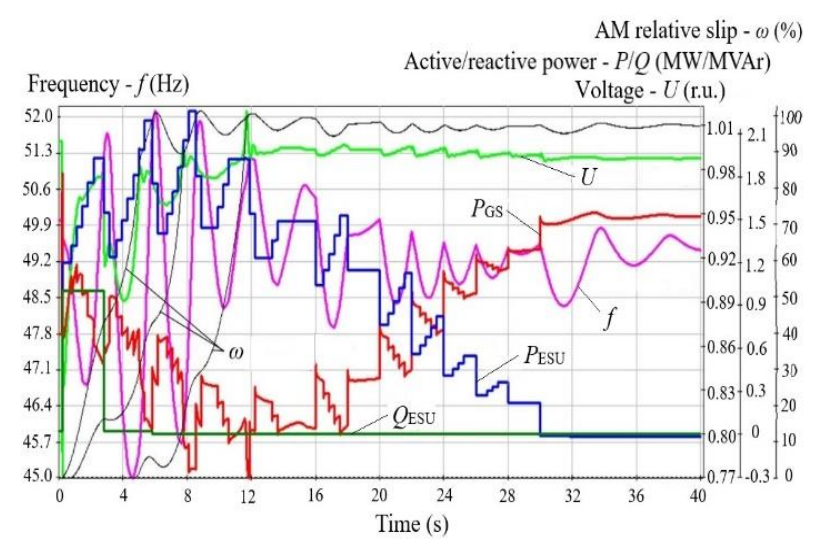

Fig. 4. Transient caused by GS load surge resulting from starting an AM group, ESU CA issued for $P$ and $Q$.

The total nominal power of these AMs equals $37.5 \%$ of the total nominal output of the GPUs, which far exceeds the capacity for starting motors in groups in islanded operation unless additional GS's are involved.

\subsection{Shedding $95 \%$ of the load at nominal initial GS load}

In this case, hazard comes from a transient characterized by frequency deviation; early in such a transient, ESU CAs are executed with total active power consumption equal to the shed load: $\Delta P_{\mathrm{ESU}}=-2.3 \mathrm{MW}$, see Figure 5 .

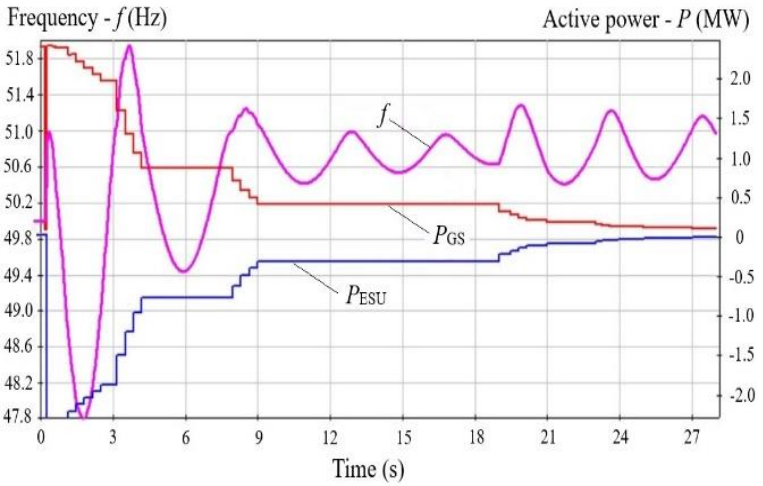

Fig. 5. Transient associated with shedding $95 \%$ of the load by an ESU CA for $P$ and $Q$.

If substantial power shedding is likely, but the GS's should remain online, then normal ESU controls should enabe the unit to keep such accumulated charge $\int P_{\mathrm{ESU}} d t$ that would provide an available power of $\pm \Delta P_{\mathrm{GS} . n o m}$.

\subsection{Three-phase SC in a DG facility-carrying microgrid}

Figure 6 shows a transient where an SC disrupts electricity delivery to a substation that carries a load of $27 \%$ of $P_{\mathrm{GS} . n o m}$, after which such delivery is automatically restored in $0.38 \mathrm{~s}$ by ALTs.

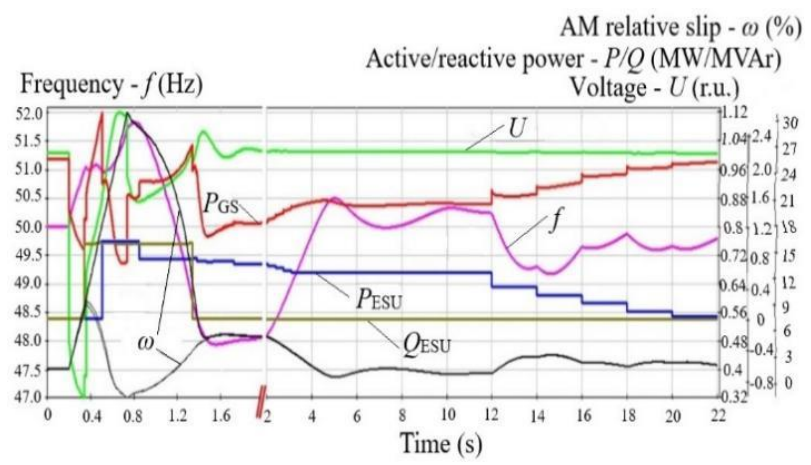

Fig. 6. Three-phase SC transient in a microgrid, ESU CAs for $P$ and $Q$.

The transient shown in Figure 6 is different from the two previous transients, as in its case, a higher-power ESU provides more efficient controls. However, there is no general pattern here, as optimal ESU CA adjustments will vary for different SC durations and significantly different load parameters. Thus, ESU CA adjustments should be optimized by analyzing the probability of SCs in the context of using standard dynamic load stability improvements.

Simulations should be used to help to define technical requirements to ESU. Table 1 presents such requirements for transients shown in Figures 4 to $6(P>$ 0 when the ESU is delivering power to the grid).

Thus, we have analyzed the effectiveness of the proposed ESU control method for extending the acceptable range of GS parameters at DG facilities; this analysis concludes that the method is suitable for DG facility-carrying microgrids. 
Table 1. Examples of ESU requirements.

\begin{tabular}{|c|c|c|c|c|}
\hline Figure & $U_{\text {nom }, \mathrm{kV}}$ & $I_{\max }, \mathrm{A}$ & $P_{\max }, \mathrm{MW}$ & $\begin{array}{c}\text { Energy } \\
\text { intensity ESU, } \\
\mathrm{MW} \cdot \mathrm{s}\end{array}$ \\
\hline 4 & 6,3 & 220 & $+2,1$ & +25 \\
\hline 5 & 6,3 & 220 & $-2,4$ & -12 \\
\hline 6 & 6,3 & 130 & $+1,0$ & +10 \\
\hline
\end{tabular}

The method effectively prevents unnecessary GS disconnections when an islanded DG facility is exposed to severe disturbances; this helps deliver electricity more reliably.

\section{Conclusions}

Foreign manufacturers configure RPs in a way that causes unnecessary GS disconnections at DG facilities when frequencies deviate shortly due to transients; this disrupts electricity delivery to critical users, which entails heavy damage.

To prevent unnecessary GS disconnections, this paper proposes the method that independently controls active $\left(\Delta P_{\mathrm{ESU}}\right)$ and reactive $\left(\Delta Q_{\mathrm{ESU}}\right)$ power output/consumption at a combined ESU, which comprises a supercapacitor and a li-ion battery.

The paper further showcases the effectiveness of controlling ESU reactive power by relay-forced GS excitation ( $\Delta Q_{\mathrm{ESU}}>0$ if $\left.U<U_{\mathrm{min}}\right)$, which means there is no need to reconfigure the ACS for all the active grid components that prevent oscillatory instability and dampen oscillations in case of short-term disturbances.

The idea is to control ESU active power:

- Early in a transient once a rapid change in generation $\left(\Delta P_{\mathrm{GS}}\right)$ has been detected by executing a $\mathrm{CA}_{\mathrm{ESU}-P}$ equal to $\Delta P_{\mathrm{ESU}}$ with a minimum lag, whereby the value of $\Delta P_{\mathrm{ESU}}$ is proportional to the frequency derivative,

- By checking frequency at equal time intervals $T$ and, if $f<f_{1}$, issuing a CA to raise $P_{\text {ESU }}$ (reduce the GS output and therefore increase the frequency) or, if $f>f_{2}$, lowering $P_{\text {ESU. }} T$ and ESU CA (power) adjustments $\left(+\Delta P_{\mathrm{ESU}},-\Delta P_{\mathrm{ESU}}\right)$ should be optimized by simulation.

In case of the worst disturbances (starting a group of motors; shedding $95 \%$ of the GS power output; a threephase SC in the microgrid), the proposed method prevents unnecessary GS disconnections while not changing the $\mathrm{CA}$ adjustments $\left(+\Delta P_{\mathrm{ESU}},-\Delta P_{\mathrm{ESU}}\right)$. Therefore, there is no need to adjust ESU CAs online for frequency deviation.

Technical requirements to ESUs should be optimized by simulating various operating situations and possible disturbances that may arise in islanded operation.

The developed method that independently controls $\Delta P_{\mathrm{ESU}}$ and $\Delta Q_{\mathrm{ESU}}$ can extend the acceptable range of GS parameters at DG facilities to prevent unnecessary GS disconnections that would otherwise be caused by shortterm frequency deviations; it therefore enables more reliable electricity delivery in an islanded microgrid.

\section{References}

1. B. Buchholz, Z. Styczynski, Smart Grids fundamentals and technologies in electricity networks (Springer Heidelberg, 2014)

2. S. Filippov, M. Dilman, P. Ilyushin, Thermal Engineering, 66 (12), 869-880 (2019)

3. M. Schifani, E. Waffenschmidt, R. Iravani, Proc. of the International Energy and Sustainability Conference, IESC (2017)

4. A. Kulikov, M. Sharygin, P. Ilyushin, Power Technology and Engineering, 53 (5), 611-617 (2020)

5. P. Ilyushin, Power Technology and Engineering, 51 (6), 713-718 (2018)

6. P. Ilyushin, A. Kulikov, A. Loskutov, Advances in Intelligent Systems and Computing, 1295, 104120 (2020)

7. S. Budi, A. Nurdiansyah, A. Lomi, Proc. of the International Sem. on Intelligent Technology and Its Applications, ISITIA (2017)

8. P. Ilyushin, O. Sukhanov, Russian Electrical Engineering, 85 (3), 133-137 (2014)

9. P. Ilyushin, A. Mokeev, V. Narovlyanskii, Power Technology and Engineering, 53 (2), 240-247 (2019)

10. P. Ilyushin, P. Berezovskiy, Energetik, 11, 3-8 (2019)

11. Z. Yao, G. Geng, Q. Jiang, IEEE Transactions on Power Systems, 32 (4), 2704-2713 (2017)

12. G. Brukhis, V. Voronin, P. Ilyushin, N. Gorshkova, Power Technology and Engineering, 46 (4), 305-311 (2012)

13. T. Bambaravanage, S. Kumarawadu, A. Rodrigo, L. Widanagama, Proc. of the IEEE Int. Conf. on Signal Processing, Computing and Control (2013)

14. K. Kilani, M. Elleuch, A. Hamida, Proc. of the 14th International Multi-Conference on Systems, Signals \& Devices, SSD (2017)

15. V. Samoylenko, P. Ilyushin, A. Pazderin, Renewable Energy and Power Quality Journal, 18, 70-75 (2020)

16. Sk. Islam, D. Sutanto, K. Muttaqi, IEEE Transactions on Power Systems, 30 (5), 25912603 (2015)

17. L. Rosman, Means and control systems in the energy sector, Overview information, 2 (1987)

18. P. Georgilakis, N. Hatziargyriou, Electric Power Systems Research, 121 (2), 89-100 (2015)

19. F. Conte, F. D'Agostino, F. Silvestro, Electric Power Systems Research, 168, 92-104 (2019)

20. A. Ehsan, Q. Yang, Applied Energy, 239 (1), 1509-1523 (2019) 